\title{
Low acoustic transmittance through a holey structure
}

\author{
J. S. Bell, " I. R. Summers, A. R. J. Murray, E. Hendry, J. R. Sambles, and A. P. Hibbins \\ Department of Physics and Astronomy, University of Exeter, Stocker Road, Exeter EX4 4QL, United Kingdom
}

(Received 22 May 2011; revised manuscript received 18 May 2012; published 27 June 2012)

\begin{abstract}
The "acoustic double fishnet" is a structure with holes running from its front to back faces, yet at a characteristic frequency it transmits very little sound. The transmittance of this structure, which is comprised of a pair of closely spaced, periodically perforated plates, is determined experimentally and analytically. The surprising acoustic properties are due to hybridization between a two-dimensional resonance within the gap between the plates, and pipe modes within the holes. At the center of the stop band the input impedance is imaginary, interpreted as a negative product of effective bulk modulus and density.
\end{abstract}

DOI: 10.1103/PhysRevB.85.214305

PACS number(s): 43.20.El, 43.20.Mv, 43.55.Ev

\section{INTRODUCTION}

Acoustic metamaterials are artificial structures, engineered on the subwavelength scale to produce novel performance in the control of sound. Significant new research in the field of acoustic metamaterials began over 10 years ago, with one of the earliest pioneering works showing that the transmittance of a cubic array of composite spheres breaks the mass-density transmission law by an order of magnitude. ${ }^{1}$ This spawned further research into fields such as acoustic cloaking ${ }^{2-4}$ and lensing. ${ }^{5-8}$ Explorations of the acoustic transmittance of perforated plates ${ }^{9-12}$ and, more recently, theoretical investigations of pairs of perforated plates ${ }^{13-15}$ have followed. One approach to characterizing the acoustic behavior of metamaterials is through "effective" values for the bulk modulus $K$ and mass density $\rho$. Several structures have been shown to possess negative values of $K$ and/or $\rho$ at characteristic frequencies. ${ }^{16-18}$

The acoustic double fishnet (ADF) structure (Fig. 1), previously analyzed by Liu et al. ${ }^{14}$ consists of two perfectly rigid plates separated by a gap $d_{\mathrm{g}}$. Each plate is of thickness $d_{\mathrm{p}}$ and is perforated by holes of radius $a$ in a square array of pitch $\Lambda$. The holes in one plate are aligned with the holes in the other. The present study provides a combined analytical and experimental exploration of the acoustic transmittance of this structure.

\section{THEORETICAL MODELS}

A modal matching method was developed to model the transmittance of the ADF. The pressure fields in regions outside the plates (region 1 on the incident side of the structure, region 3 between the plates, and region 5 on the transmitted side) are matched to the fields in the holes in the two plates (regions 2 and 4). In region 1 the pressure field is a sum of the normally incident unit plane wave (wave number $k_{0}$ ) and a twodimensional Fourier-Floquet expansion of diffracted orders with wave vectors $\left(k_{x}^{m_{1}, m_{2}}, k_{y}^{m_{1}, m_{2}}, k_{z}^{m_{1}, m_{2}}\right)$. The pressure field inside the holes of the first and second plates (regions 2 and 4 ) is expanded in terms of the first-order acoustic mode of the cavities. Similar definitions for the pressure fields in regions 3-5 give the definitions for pressure fields in all five regions:

$$
\begin{aligned}
P_{1}(x, y, z)= & \psi^{0,0} \exp \left(i k_{z}^{0,0} z\right) \\
& +\sum_{m_{1}, m_{2}} R^{m_{1}, m_{2}} \psi^{m_{1}, m_{2}} \exp \left(-i k_{z}^{m_{1}, m_{2}} z\right),
\end{aligned}
$$

$$
\begin{aligned}
P_{2}(x, y, z)= & A_{1} \exp \left(i k_{0} z\right)+A_{2} \exp \left(-i k_{0} z\right), \\
P_{3}(x, y, z)= & \sum_{m_{1}, m_{2}} B_{1}^{m_{1}, m_{2}} \psi^{m_{1}, m_{2}} \exp \left(i k_{z}^{m_{1}, m_{2}} z\right) \\
& +\sum_{m_{1}, m_{2}} B_{2}^{m_{1}, m_{2}} \psi^{m_{1}, m_{2}} \exp \left(-i k_{z}^{m_{1}, m_{2}} z\right), \\
P_{4}(x, y, z)= & C_{1} \exp \left(i k_{0} z\right)+C_{2} \exp \left(-i k_{0} z\right), \\
P_{5}(x, y, z)= & \sum_{m_{1}, m_{2}} T^{m_{1}, m_{2}} \psi^{m_{1}, m_{2}} \exp \left(i k_{z}^{m_{1}, m_{2}} z\right),
\end{aligned}
$$

where

$\psi^{m_{1}, m_{2}}=\exp \left[i\left(k_{x}+\frac{2 m_{1} \pi}{\Lambda}\right) x\right] \exp \left[i\left(k_{y}+\frac{2 m_{2} \pi}{\Lambda}\right) y\right]$.

Note that we omit the time-dependent components of the fields. Integer pairs $\left(m_{1}, m_{2}\right)$ denote the diffracted orders of the hole arrays of pitch $\Lambda$ and the factors $R^{m_{1}, m_{2}}$ and $T^{m_{1}, m_{2}}$ describe the complex field reflection and transmission coefficients for the pressure field. The $z$ component of the wave vectors in the Fourier expansions can be written as

$$
k_{z}^{m_{1}, m_{2}}=\sqrt{k_{0}^{2}-\left(k_{x}+\frac{2 m_{1} \pi}{\Lambda}\right)^{2}-\left(k_{y}+\frac{2 m_{2} \pi}{\Lambda}\right)^{2}} .
$$

In order to eliminate the unknown sets $A, B$, and $C$, we exploit the continuity conditions of pressure fields at the boundaries: The pressure field must be continuous at the entrances and exits of the holes, while the normal component of the pressure gradient must be continuous over the entire unit cell. These boundary conditions give two continuity equations for each interface of the structure. Solving these continuity equations for $R$ and $T$, eliminating the unknowns, yields zero-order transmission functions

$$
T^{0,0}=\frac{k_{0}}{\Lambda^{2} k_{z}^{0,0}} \frac{4 Q_{-}^{0,0} Q_{+}^{0,0}}{\exp \left(-2 i k_{0} d_{\mathrm{p}}\right) \frac{\left(\pi a^{2}+S_{1}\right)^{2}}{\pi a^{2}}-\exp \left(2 i k_{0} d_{\mathrm{p}}\right) \frac{\left(\pi a^{2}+S_{1}\right)^{2}}{\pi a^{2}}}
$$

for a single fishnet and

$$
T^{0,0}=\frac{k_{0}}{\Lambda^{2} k_{z}^{0,0}} \frac{4 Q_{-}^{0,0} Q_{+}^{0,0}}{D},
$$




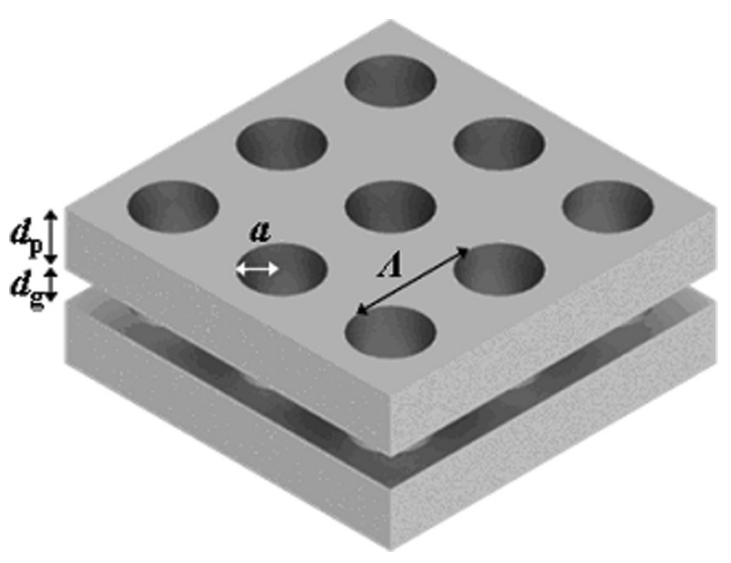

FIG. 1. Schematic of nine unit cells of the acoustic double fishnet (ADF) structure. This is comprised of a pair of identical plates of thickness $d_{\mathrm{p}}$ drilled with a square array of holes of radius $a$ and pitch $\Lambda$ (in experiments $a=1.2 \mathrm{~mm}$ and $\Lambda=8 \mathrm{~mm}$ ), separated by a gap $d_{\mathrm{g}}$ which is small compared to the wavelength of the normally incident planar sound waves.

where

$$
\begin{aligned}
D= & \exp \left(-2 i k_{0} d_{\mathrm{p}}\right) \frac{\left(\pi a^{2}+S_{1}\right)^{2}\left[\left(\pi a^{2}+S_{2}\right)^{2}-S_{3}^{2}\right]}{2 \pi^{2} a^{4} S_{3}} \\
& +\exp \left(2 i k_{0} d_{\text {plate }}\right) \frac{\left(\pi a^{2}-S_{1}\right)^{2}\left[\left(\pi a^{2}-S_{2}\right)^{2}-S_{3}^{2}\right]}{2 \pi^{2} a^{4} S_{3}} \\
& -2 \frac{\left(\pi^{2} a^{4}-S_{1}^{2}\right)\left(\pi^{2} a^{4}-S_{2}^{2}+S_{3}^{2}\right)}{2 \pi^{2} a^{4} S_{3}}
\end{aligned}
$$

for the $\mathrm{ADF}$ structure, where

$$
\begin{aligned}
& S_{1}=\sum_{m_{1}, m_{2}} \frac{k_{0}}{\Lambda^{2} k_{z}^{m_{1}, m_{2}}} Q_{-}^{m_{1}, m_{2}} Q_{+}^{m_{1}, m_{2}}, \\
& S_{2}=\sum_{m_{1}, m_{2}} i \cot \left(k_{z}^{m_{1}, m_{2}} d_{\text {gap }}\right) \frac{k_{0}}{\Lambda^{2} k_{z}^{m_{1}, m_{2}}} Q_{-}^{m_{1}, m_{2}} Q_{+}^{m_{1}, m_{2}}, \\
& S_{3}=\sum_{m_{1}, m_{2}} i \csc \left(k_{z}^{m_{1}, m_{2}} d_{\text {gap }}\right) \frac{k_{0}}{\Lambda^{2} k_{z}^{m_{1}, m_{2}}} Q_{-}^{m_{1}, m_{2}} Q_{+}^{m_{1}, m_{2}} .
\end{aligned}
$$

The overlap integrals $Q$ between the $\left(m_{1}, m_{2}\right)$ th diffracted order and the acoustic cavity modes are given by

$$
\begin{aligned}
Q_{ \pm}^{m_{1}, m_{2}}= & \int_{S} \exp \left[ \pm i\left(k_{x}+\frac{2 \pi m_{1}}{\Lambda}\right) x\right] \\
& \times \exp \left[ \pm i\left(k_{y}+\frac{2 \pi m_{2}}{\Lambda}\right) y\right] d S,
\end{aligned}
$$

where the surface integral is over the circular entrance to the cylindrical cavity.

Figure 2 (bottom panel) shows the predicted amplitude transmission coefficient of the ADF structure as a function of frequency and plate thickness. The peaks in transmission can be attributed to the resonance frequencies of the structure.

To interpret the results of the model, consider a unit cell of the square array, centered on two aligned holes (see Fig. 1, which shows nine unit cells). The two holes may be considered as forming a single open-ended pipe with a resonant side branch (i.e., a gap) at its midpoint. This pipe supports a set of standing-wave modes. The even-order modes have a pressure
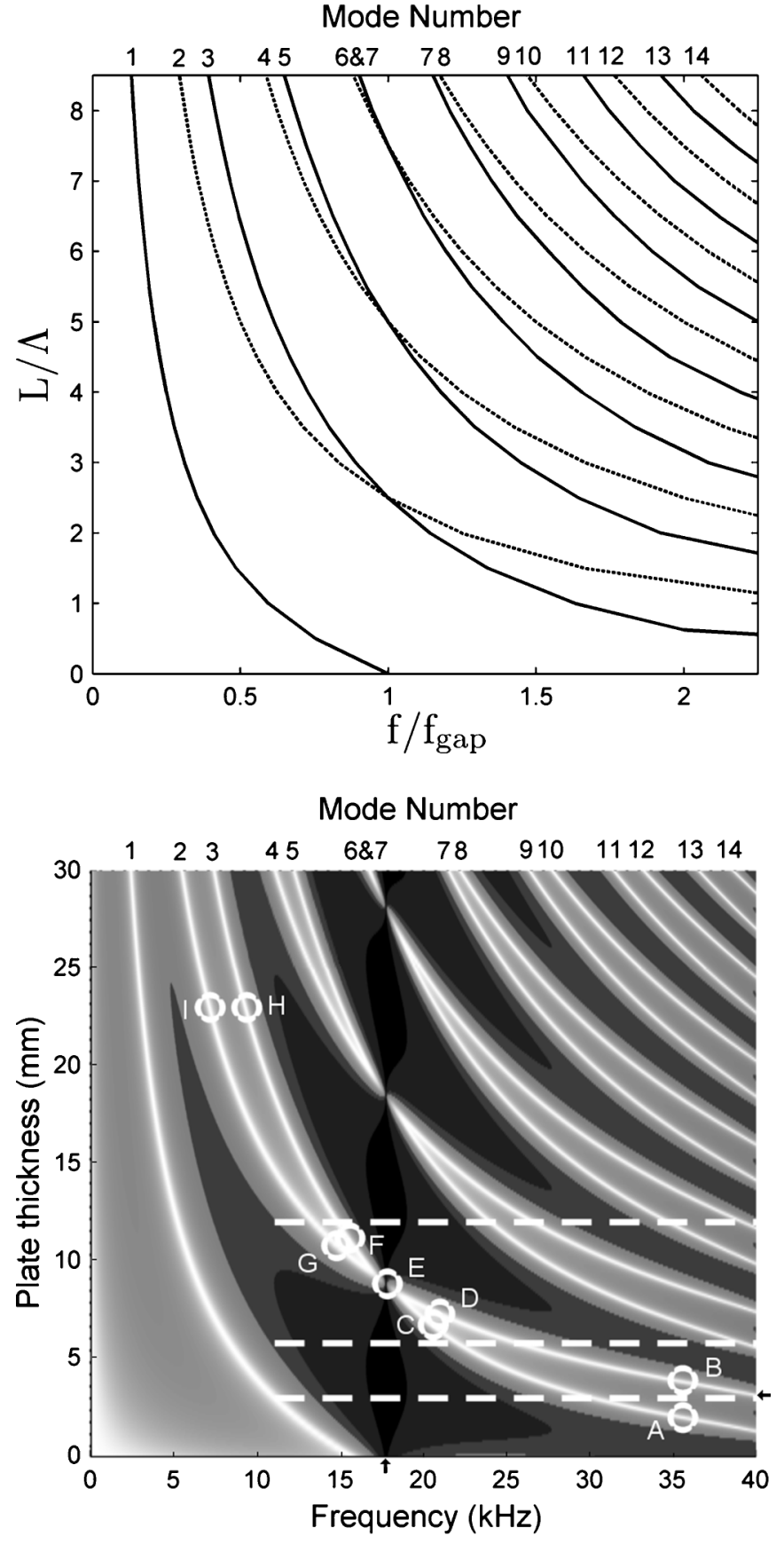

FIG. 2. (Top) Resonance frequencies of the ADF structure given by Eqs. (6) and (7). Odd-order modes are solid lines and even-order modes are dashed. (Bottom) Amplitude transmission coefficient as a function of frequency for varying plate thickness $d_{\mathrm{p}}$ obtained using Eqs. (1)-(5) with $a=1.2 \mathrm{~mm}, \Lambda=8 \mathrm{~mm}$, and $d_{\mathrm{g}}=0.47 \mathrm{~mm}$. The black regions correspond to transmission of less than $1 \%$, and the two shades of dark gray correspond to transmission of less than $5 \%$ and $10 \%$. The dashed white lines indicate the three plate thicknesses for which experimental data were obtained, and the points labeled A to I correspond to the pressure profiles in Fig. 3. The arrow on the horizontal axis of the bottom panel indicates the frequency that corresponds to $f / f_{\text {gap }}=1$; the arrow on the vertical axis of the bottom panel indicates the plate thickness that corresponds to $L / \Lambda=1$.

node halfway along the pipe and are not significantly affected by the presence of the gap. However, the odd-order modes have 
a pressure antinode halfway along the pipe and so are shifted in frequency (compared to the no-gap case, i.e., a uniform pipe) due to coupling to the mode in the gap region. The extent of frequency shift for the odd-order modes is determined by the acoustic input impedance of the gap, which exhibits a minimum at a resonance frequency determined by the pitch $\Lambda$ of the square array.

The frequency of this "gap resonance" might be naively estimated as $f_{\text {gap }} \approx c / 2 \Lambda$ due to continuity requirements in the fields at the edges of each unit cell, where $c$ is the speed of sound in air. A more accurate expression $f_{\text {gap }} \approx c / 2.5 \Lambda$ can be obtained by considering the principal mode of the part of the gap region associated with a unit cell, i.e., the lowest-frequency mode of a square zone of the gap region, centered on a hole (see Fig. 1).

The resonance frequencies of the even-order pipe modes are given by

$$
f=\frac{n c}{2 L},
$$

where $n$ is an even integer, $c$ is the speed of sound, and $L$ is the effective pipe length (i.e., twice the plate thickness $d_{\mathrm{p}}$, plus a small allowance for end corrections and for the plate separation $d_{\mathrm{g}}$ ).

For the odd-order modes, the profiles for volume flow rate along the pipe are sinusoidal within each half of the pipe, but include a discontinuity at the midpoint because of volume flow from the pipe into the gap region. (Corresponding discussion of pressure profiles is included below, in relation to Fig. 3.) This results in a shift of frequency for these modes, compared to the no-gap case, the extent of which depends on the acoustic input impedance of the gap (as mentioned above) and can be calculated by considering the conservation of volume flow within the system. This calculation is summarized in the

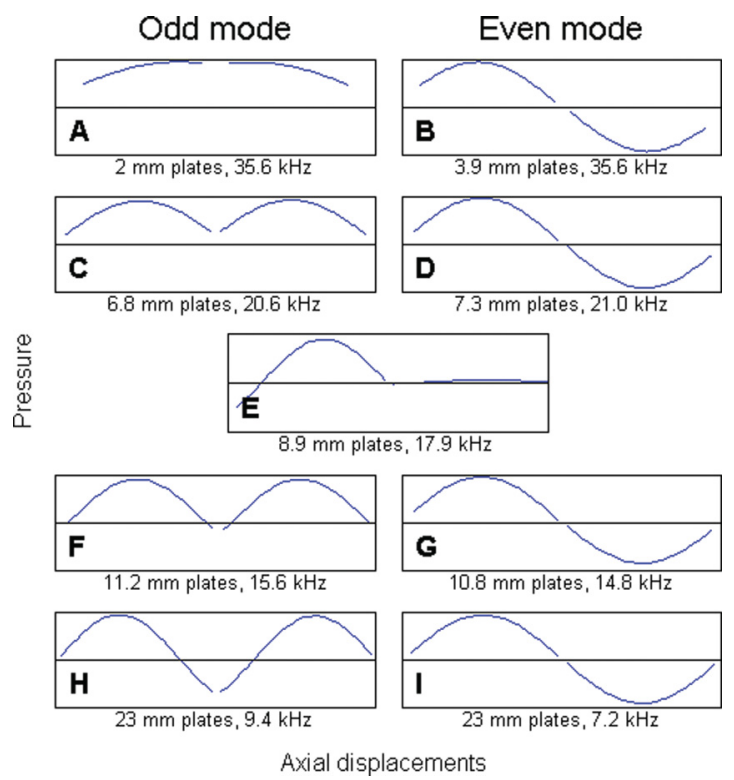

FIG. 3. (Color online) Pressure profiles of the resonant peaks labeled in Fig. 2. The shape of the even-order mode remains essentially unchanged as the plate thickness varies; however, the odd-order mode changes due to leakage into the gap region.
Appendix and results in the following relation:

$$
f=-\frac{c}{\pi L} \tan ^{-1}\left(\frac{2 X}{Z}\right),
$$

where $Z$ is the acoustic impedance for a traveling wave in the pipe, and $X$ is the input reactance of the gap, which exhibits a resonance minimum at the frequency $f_{\text {gap }}$. The predicted resonance frequencies for both the even-order and odd-order modes (normalized to the gap-resonance frequency $f_{\text {gap }}$ ) are shown in Fig. 2 (top panel) over a range of values for $L$ (normalized to the hole spacing $\Lambda$ ), i.e., over a range of plate thicknesses $d_{\mathrm{p}}$.

Comparison of the two methods for finding the resonance frequencies of the ADF shows a clear agreement, despite the approximations involved in the analytical calculation. This suggests that the insights provided by the analytical calculation into the natures of the various modes are also applicable to the transmission peaks calculated using the modal matching method. As the frequency approaches the gap-resonance frequency $f_{\text {gap }}$, either from above or below, the odd-order modes (which are the modes affected by coupling into the gap region) move towards the even-order modes, and at the gap-resonance frequency the odd-order modes coincide with the even-order modes.

As mentioned above, and elaborated upon in the Appendix, the acoustic input impedance of the gap exhibits a resonance minimum at the gap-resonance frequency $f_{\text {gap }}$. At frequencies close to $f_{\text {gap }}$, the acoustic input impedance of the gap is small and, due to the impedance mismatch at the interface between the holes and the gap, transmission through the structure is low and reflection from the structure is high. At the gap-resonance frequency, transmission shows a minimum and reflection shows a maximum - this is associated with the imaginary part of the input impedance of the structure becoming dominant at this frequency. If this impedance is expressed as $Z_{\text {in }}=(K \rho)^{1 / 2}$, where $K$ is an effective bulk modulus and $\rho$ is an effective density, a purely imaginary value at the gap-resonance frequency implies a negative product of $K$ and $\rho$. (An approach based on input impedance has been chosen here, so as to best illustrate the underlying physical behavior-with such an approach it is not possible to consider $K$ and $\rho$ separately.)

Further results from the modal-matching analysis are given in Fig. 3, in the form of axial pressure profiles within the pairs of holes in the structure. Profiles are shown for the case when the frequency of the incident wave matches an even-order pipe mode (the second mode, calculated at the points B, D, E, G, I indicated in Fig. 2, bottom panel) and the case when the frequency of the incident wave matches an odd-order pipe mode (the first mode, calculated at points A, C, E, which becomes the third mode, calculated at points $\mathrm{E}, \mathrm{F}, \mathrm{H})$. At point $\mathrm{E}$, at the frequency of the gap resonance, the modes coincide and the pressure profile is the sum of the antisymmetric second mode and the symmetric first/third mode; the odd and even modes coexist and destructively interfere, resulting in no transmission through the structure. The even-order modes, which do not couple to the gap resonance, have a sinusoidal profile with corresponding frequencies in an even-harmonic series, as indicated by Eq. (2). The odd-order modes, which do couple to the gap resonance, have profiles that include 
a "kink" that derives from the leakage of volume flow into the gap. Because the odd-order profiles are not sinusoidal, the resonance wavelengths are not in simple integer ratios to the effective pipe length. Hence, the mode frequencies are shifted from the odd-harmonic series of the no-gap case, as shown by Eq. (3). The shift in frequency is upwards for frequencies above the gap-resonance frequency and downwards for frequencies below the gap-resonance frequency.

\section{EXPERIMENT AND RESULTS}

The acoustic transmittance through three ADF structures has been measured experimentally using a collimated acoustic beam arrangement with two spherical mirrors, radius of curvature of $2 \mathrm{~m}$, one creating an approximately plane wave front at the sample and the other focusing the transmitted signal onto a detector microphone. The three ADF structures consist of pairs of square Perspex (Lucite) plates with side $200 \mathrm{~mm}$ and thicknesses $3.0,5.9$, or $12.0 \mathrm{~mm}$ (indicated by the white dashed lines in Fig. 2 (bottom). Each plate has holes of radii $1.2 \mathrm{~mm}$ arranged in a square array of pitch $8 \mathrm{~mm}$. The plates comprising each pair were clamped together with a separation of either 0.47 or $0.94 \mathrm{~mm}$, and arranged so that the holes were accurately aligned. The transmission data are normalized to the signal in the absence of a sample.

Figure 4 shows the experimentally determined transmittance of each of the three pairs of plates separated by gaps of 0.47 or $0.94 \mathrm{~mm}$. Data were recorded between 10 and 42 $\mathrm{kHz}$, the upper limit of the range corresponding to the onset of diffraction. The data are clearly in good agreement with the predictions from the modal matching method. (The frequency sweeps for each plate thickness and $d_{\mathrm{g}}=0.47 \mathrm{~mm}$ are represented by the dotted lines shown in Fig. 2, bottom panel.) The transmittance drops to around $-35 \mathrm{~dB}$ or less (around $2 \%$ in amplitude) at the center of the stop band for all six structures. This residual transmission may be attributed to some acoustic leakage around the sample. The experimental transmittance

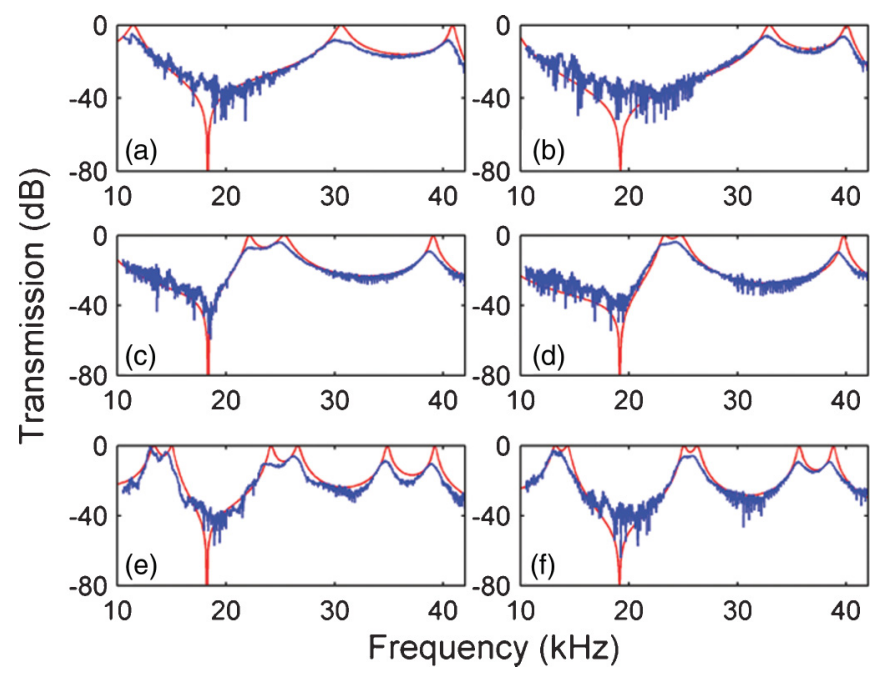

FIG. 4. (Color online) Experimentally determined transmittance (blue) and modal-matching theory (red) for ADFs with the following plate thickness $d_{\mathrm{p}}$ and gap $d_{\mathrm{g}}$ : (a) $3 \mathrm{~mm}$ and $0.47 \mathrm{~mm}$, (b) $3 \mathrm{~mm}$ and $0.94 \mathrm{~mm}$, (c) $5.9 \mathrm{~mm}$ and $0.47 \mathrm{~mm}$, (d) $5.9 \mathrm{~mm}$ and $0.94 \mathrm{~mm}$, (e) $12 \mathrm{~mm}$ and $0.47 \mathrm{~mm}$, (f) $12 \mathrm{~mm}$ and $0.94 \mathrm{~mm}$.

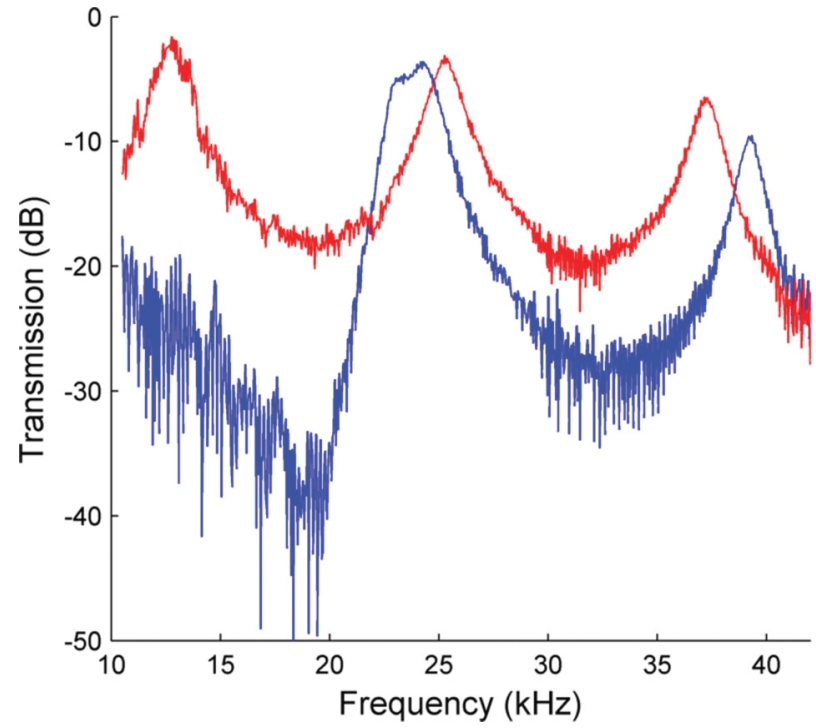

FIG. 5. (Color online) Experimentally determined transmittance of the ADF with $d_{\mathrm{p}}=3 \mathrm{~mm}$ (blue) and a single $5.9 \mathrm{~mm}$ perforated plate (red).

does not reach unity at the resonances as predicted by the modeling. This is most likely because the theoretical model does not include the effects of viscous loss.

Figure 5 compares the experimentally determined transmittance of the ADF comprising 3-mm-thick plates separated by a gap of $0.47 \mathrm{~mm}$, with that of a single $5.9-\mathrm{mm}$ perforated plate of the same pitch and hole diameter. The transmittance of the $\mathrm{ADF}$ at the gap-resonance frequency is around $15 \mathrm{~dB}$ less than the lowest value found for the single plate.

The dispersion behavior of the modes supported by the ADF was also determined experimentally. The apparatus was comprised of the same spherical mirror assembly described earlier, with the sample mounted on an adjustable turntable and behind an aperture. The angle-dependent transmittance is presented as a dispersion plot in Fig. 6 (left). The theoretical dispersion behavior in Fig. 6 (right) was determined using the modal matching method, and shows compelling agreement

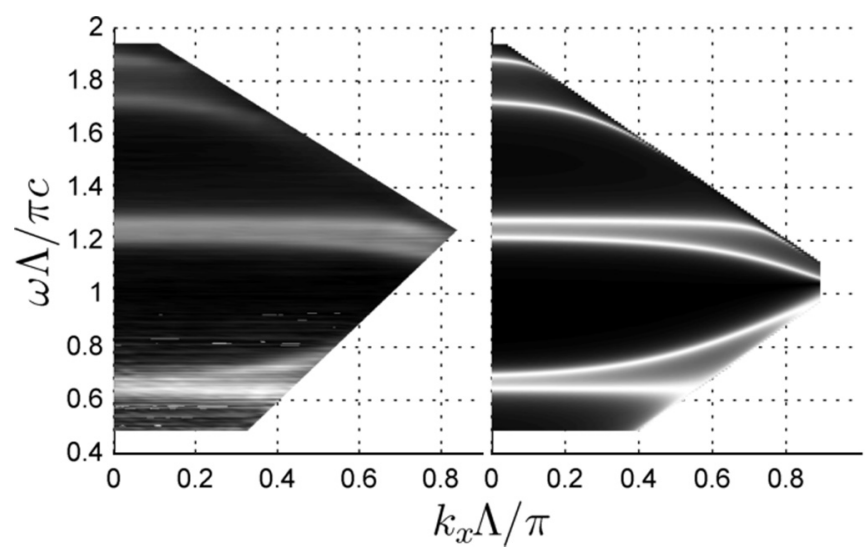

FIG. 6. (Left) Experimentally determined dispersion properties of the ADF with $d_{\mathrm{p}}=12 \mathrm{~mm}$ separated by a gap $d_{\mathrm{g}}=0.47 \mathrm{~mm}$. The graph is incomplete as data could only be obtained between normal incidence and an azimuth angle of $40^{\circ}$. (Right) Theoretical dispersion properties of the $\mathrm{ADF}$ with the same configuration as the experiment. 
with both the experimental data and the predictions of Christensen et al. ${ }^{13}$

According to the modeling for normal incidence, for a plate thickness $d_{\mathrm{p}}=8.9 \mathrm{~mm}$ a transmittance of less than $-20 \mathrm{~dB}$ (i.e., $10 \%$ in amplitude, $1 \%$ in intensity) is obtained over a frequency range of 9-27 kHz. This is a remarkably broad stop band considering the relative simplicity of the structure. This stop band is readily tuned by adjusting the thickness of the plates, the periodicity of the hole array, and the gap width. The frequency range over which strong attenuation is achieved is dependent upon the $Q$ factor of the pipe resonances, ${ }^{9,10}$ and to attain efficient attenuation over a substantial range, holes that are small in relation to the plate thickness are required. Holes can be made as small as fabrication allows as, unlike the electromagnetic case in metal plates, there is no cutoff frequency for transmission. ${ }^{11}$

\section{CONCLUSION}

The acoustic transmittance of the ADF structure has been investigated both analytically and experimentally and good agreement found. The structure has holes running from front to back but, at the center of the stop band, acoustic transmission is observed to be less than $-35 \mathrm{~dB}$, i.e., less than $2 \%$ in amplitude. This is a surprising result, and could be utilized in situations where air flow or unhindered line of sight is important. (In practice, a solid plate in air is likely to have a lower transmittance than the ADF in air, because of viscous losses, imperfections, etc., in the ADF structure). The low transmission at the center of the stop band is characterized by a purely imaginary input impedance, which can be associated with a negative product of effective bulk modulus and density - equivalent to an infinite tunnel barrier, but produced by a structure of finite thickness.

\section{ACKNOWLEDGMENTS}

The authors would like to thank the workshop technicians Nick Cole, Kevyn White, and Matt Wears for expert manufacture of the experimental apparatus. This work was made possible by funding from the EPSRC and Sonardyne International.

\section{APPENDIX: NORMAL-MODE FREQUENCIES OF AN OPEN-ENDED PIPE WITH A RESONANT SIDE BRANCH AT ITS MIDPOINT}

Consider an open-ended pipe with cross section $S$ and effective length $L$ (corrected for end effects), running in the $x$ direction from $x=-L / 2$ to $x=+L / 2$. The pipe has a resonant side branch at its midpoint, $x=0$. Assume the entrance to the side branch has negligible width in the $x$ direction. (The resonant side branch corresponds to the gap resonance described in the paper, and the pipe corresponds to two aligned holes.)

\section{Even-order modes}

The even-order pipe modes have a pressure node at $x=0$ and so do not couple to the side branch. Hence the resonance frequencies of the even-order modes are the same as for an open-ended pipe with no side branch, given by

$$
f=\frac{n c}{2 L},
$$

where $n$ is an even integer and $c$ is the speed of sound.

\section{Odd-order modes}

For the odd-order modes the acoustic pressure has nodes at $x=-L / 2$ and $x=+L / 2$ and is symmetric about the point $x=0$. In the region $0 \leqslant x \leqslant L / 2$ the acoustic pressure can be represented by the standing wave

$$
p_{1}(x, t)=D[\exp (i 2 \pi f t)] \sin \left[\left(\frac{2 \pi f}{c}\right)\left(x-\frac{L}{2}\right)\right],
$$

where $D$ is a constant and $f$ is the mode frequency. The corresponding expression for the associated standing wave of volume velocity in the region $0 \leqslant x \leqslant L / 2$ is

$U_{1}(x, t)=i \frac{D}{Z}[\exp (i 2 \pi f t)] \cos \left[\left(\frac{2 \pi f}{c}\right)\left(x-\frac{L}{2}\right)\right]$,

where $Z$ is the acoustic impedance for a traveling wave in the pipe. [Volume velocity is defined in the positive $x$ direction. Equation (A3) may be obtained from Eq. (A2) using the continuity equation $\frac{\partial p_{1}}{\partial t}=-Z c \frac{\partial U_{1}}{\partial x}$.]

At $x=0$, Eqs. (A2) and (A3) give

$$
p_{1}(0, t)=-D[\exp (i 2 \pi f t)] \sin \left(\frac{\pi f L}{c}\right)
$$

and

$$
U_{1}(0, t)=i \frac{D}{Z}[\exp (i 2 \pi f t)] \cos \left(\frac{\pi f L}{c}\right)
$$

By a similar argument (considering the symmetry about $x=0)$, the standing wave $p_{2}(x, t), U_{2}(x, t)$ in the region $-L / 2 \leqslant x \leqslant 0$ gives, at $x=0$,

$$
p_{2}(0, t)=-D[\exp (i 2 \pi f t)] \sin \left(\frac{\pi f L}{c}\right)
$$

and

$$
U_{2}(0, t)=-i \frac{D}{Z}[\exp (i 2 \pi f t)] \cos \left(\frac{\pi f L}{c}\right) .
$$

The pressure $p_{3}(t)$ at the entrance to the side branch $(x=0)$ can be obtained from Eq. (A4) [or equivalently from Eq. (A6)], i.e.,

$$
p_{3}(t)=-D[\exp (i 2 \pi f t)] \sin \left(\frac{\pi f L}{c}\right) .
$$

For reasons of continuity, the volume velocity $U_{3}(t)$ into the side branch is the difference between the expressions in Eqs. (A7) and (A5), i.e.,

$$
\begin{aligned}
U_{3}(t)= & U_{2}(0, t)-U_{1}(0, t)=-i \frac{2 D}{Z}[\exp (i 2 \pi f t)] \\
& \times \cos \left(\frac{\pi f L}{c}\right) .
\end{aligned}
$$

Additionally, $p_{3}(t)$ and $U_{3}(t)$ are related by the acoustic input impedance of the side branch which, assuming a lossless 
side branch, is purely imaginary. Using Eqs. (A8) and (A9), this relation can be written as

$$
\frac{p_{3}(t)}{U_{3}(t)}=\frac{Z}{2 i} \tan \left(\frac{\pi f L}{c}\right)=i X,
$$

where $X$ is the input reactance of the side branch.

Equation (A10) may be rearranged to give an expression for the frequencies of the odd-order modes:

$$
f=-\frac{c}{\pi L} \tan ^{-1}\left(\frac{2 X}{Z}\right) .
$$

(Note that $Z$ is independent of $f$, but $X$ is a function of $f$; see below.)

For a resonant side branch with a resonance frequency $f_{\text {gap }}$, the input reactance at frequency $f$ can be written as

$$
X=-\frac{Z S}{\alpha} \cot \left(\frac{\pi}{2} \frac{f}{f_{\text {gap }}}\right),
$$

where $\alpha$ is the cross section of the entrance to the side branch. [This expression is easily derived for a resonant side branch in the form of a closed pipe of uniform cross section $\alpha$; for a resonant side branch of a different geometry, Eq. (A12) is only an approximation. Note that the input reactance $X$ goes to zero at the resonance frequency $f_{\text {gap }}$.]

Equations (A11) and (A12) can be combined to give

$$
\tan \left(\frac{\pi f L}{c}\right)=\frac{2 S}{\alpha} \cot \left(\frac{\pi}{2} \frac{f}{f_{\text {gap }}}\right),
$$

which can be solved numerically to obtain values for the frequencies $f$ of the odd-order normal modes. [The solutions shown in the paper, for the case where the resonant side branch corresponds to the gap resonance and hence Eq. (A12) is an approximation, were obtained using an empirical value of 1.25 for the constant $2 S / \alpha$.]
*Corresponding author: j.s.bell@exeter.ac.uk

${ }^{1}$ Z. Liu, X. Zhang, Y. Mao, Y. Y. Zhu, Z. Yang, C. T. Chan, and P. Sheng, Science 289, 1734 (2000).

${ }^{2}$ H. Chen and C. Chan, Appl. Phys. Lett. 91, 183518 (2007).

${ }^{3}$ S. A. Cummer, B. I. Popa, D. Schurig, D. R. Smith, J. Pendry, M. Rahm, and A. Starr, Phys. Rev. Lett. 100, 024301 (2008).

${ }^{4}$ S. A. Cummer and D. Schurig, New J. Phys. 9, 45 (2007).

${ }^{5}$ M. Ambati, N. Fang, C. Sun, and X. Zhang, Phys. Rev. B 75, 195447 (2007).

${ }^{6}$ F. Cervera, L. Sanchis, J. V. Sánchez-Pérez, R. Martínez-Sala, C. Rubio, F. Meseguer, C. Lopez, D. Caballero, and J. SánchezDehesa, Phys. Rev. Lett. 88, 023902 (2001).

${ }^{7}$ S. Guenneau, A. Movchan, G. Pétursson, and S. A. Ramakrishna, New J. Phys. 9, 399 (2007).

${ }^{8}$ D. Torrent and J. Sánchez-Dehesa, New J. Phys. 9, 323 (2007).

${ }^{9}$ H. Estrada, P. Candelas, A. Uris, F. Belmar, F. J. Garcia de Abajo, and F. Meseguer, Phys. Rev. Lett. 101, 084302 (2008).
${ }^{10}$ H. Estrada, P. Candelas, A. Uris, F. Belmar, F. Meseguer, and F. J. Garcia de Abajo, Appl. Phys. Lett. 93, 011907 (2008).

${ }^{11}$ B. Hou, J. Mei, M. Ke, Z. Liu, J. Shi, and W. Wen, J. Appl. Phys. 104, 014909 (2008).

${ }^{12}$ B. Hou, J. Mei, M. Ke, W. Wen, Z. Liu, J. Shi, and P. Sheng, Phys. Rev. B 76, 054303 (2007).

${ }^{13}$ J. Christensen, L. Martin-Moreno, and F. J. Garcia-Vidal, Appl. Phys. Lett. 97, 134106 (2010).

${ }^{14}$ F. Liu, F. Cai, Y. Ding, and Z. Liu, Appl. Phys. Lett. 92, 103504 (2008).

${ }^{15}$ Z. Liu and G. Jin, J. Phys.: Condens. Matter 22, 305003 (2010).

${ }^{16}$ Y. Ding, Z. Liu, C. Qiu, and J. Shi, Phys. Rev. Lett. 99, 93904 (2007).

${ }^{17}$ S. H. Lee, C. M. Park, Y. M. Seo, Z. G. Wang, and C. K. Kim, Phys. Rev. Lett. 104, 054301 (2010).

${ }^{18}$ N. Fang, D. Xi, J. Xu, M. Ambati, W. Srituravanich, C. Sun, and X. Zhang, Nat. Mater. 5, 452 (2006). 